\title{
Nuclear Track Detectors for Relativistic Nuclear Fragmentation Studies: Comparison with Other Competitive Techniques
}

\author{
Mukhtar Ahmed Rana ${ }^{1,2,3^{*}}$, Gul Sher ${ }^{4}$, Shahid Manzoor ${ }^{5}$, Fariha Malik ${ }^{6}$, Kanwal Naz ${ }^{7}$ \\ ${ }^{1}$ The Pakistan Strategist Company, An SPD Project, PINSTECH-Admin Blk., Pakistan Institute of Nuclear Science and Technology \\ (PINSTECH), Islamabad, Pakistan \\ ${ }^{2}$ Physics Department, University of Balauchistan, Quetta, Pakistan \\ ${ }^{3}$ National Tokamak Plasma and Fusion Project, NILOP, PAEC, Islamabad, Pakistan \\ ${ }^{4}$ Materials Division, Directorate of Technology, PINSTECH, Islamabad, Pakistan \\ ${ }^{5}$ Physics Department, COMSATS Institute of Information Technology (CIIT), \\ Islamabad Campus, Islamabad, Pakistan \\ ${ }^{6}$ Isotope Applications Division, PINSTECH, Islamabad, Pakistan \\ ${ }^{7}$ Informatics Complex, H-8, Islamabad, Pakistan \\ Email: *marana@alumni.nus.edu.sg,marana@pinstech.org.pk
}

Received December 4, 2012; revised January 7, 2013; accepted January 20, 2013

Copyright (C) 2013 Mukhtar Ahmed Rana et al. This is an open access article distributed under the Creative Commons Attribution License, which permits unrestricted use, distribution, and reproduction in any medium, provided the original work is properly cited.

\begin{abstract}
The potential of the high resolution nuclear track detector (NTD) CR-39 is examined carefully for the measurement of relativistic nuclear projectile fragmentation cross sections and studies of related processes using the experience of many years of such measurements. The charge resolution and the charge resolving power of CR-39 detectors for the measurements of $158 \mathrm{~A} \mathrm{GeV}{ }^{207} \mathrm{~Pb}$ projectiles and their fragments are presented. Exposures of target-detector stacks, the chemical etching procedure and the nuclear track measurements are described in detail discussing precautions and possible errors. The procedures discussed are also valid for other NTDs. A comparison with electronic active detectors is also made considering important detection and measurement aspects. An experimental design proposing the co-use of NTDs with in-use active detectors is described.
\end{abstract}

Keywords: Nuclear Track Detection Methodology (NTDM); CR-39 Detectors; Nuclear Fragmentation; Charge Resolution; Fragmentation Cross Sections

\section{Introduction}

Fragmentation properties of shielding materials can affect protection of astronauts seriously. Radiations have also an adverse effect on microelectronic devices employed in the control systems of high altitude commercial aviation aircrafts and spacecrafts, leading to the failure of such devices [1]. Nuclear fragmentation cross sections are crucial to issues of radiation transport through shielding materials and resulting shielding effect [2]. Nuclear fragmentation cross sections also give information about collision dynamics of high energy heavy nuclei like lead and uranium. Various techniques [3-8] have been employed for studies of nuclear fragmentation and other reactions, including solid state nuclear track detector (NTD) [9-18]. Here, we discuss experimental meth-

"Corresponding author. ods and related problems for the measurement of fragmentation cross sections using NTDs. The present paper is based on the results from several investigations on the methodology of nuclear tracks [19-21] and the use of NTDs in nuclear fragmentation studies [22-25]. Present paper addresses two issues: 1) Concise description of experiments to detect fragments of exotic nuclear reaction with CR-39 and other nuclear track detectors and 2) evaluation of experimental methods for the measurement of relativistic charged particles by passive and active detectors. The study of such processes is important for fundamental physics reasons and technical applications in fusion plasma, dosimetry in space and heavy ion therapy. Due to these reasons, the present study is interesting for several scientists in nuclear physics and their application. 


\section{High Sensitivity High Resolution Detector CR-39}

An NTD is a solid material (normally plastic, glass or a crystal), when exposed to a single charged radiation, a damaged trail of diameter $2-10 \mathrm{~nm}$ is produced which, through chemical etching, can be amplified to microscopically observable conical shapes. The main advantages over other radiation detectors are the detailed information available on individual particles, the persistence of the tracks allowing measurements to be made over long periods of time, and the simple, cheap and robust construction of the detector. A material commonly known and used as an NTD is CR-39. Its chemical name is polyallyl diglycol carbonate (PADC). It is a transparent and rigid plastic material with the chemical formula $\mathrm{C}_{12} \mathrm{H}_{18} \mathrm{O}_{7}$. Etching is usually performed in solutions of caustic alkalis such as sodium or potassium hydroxide at temperatures of $60^{\circ} \mathrm{C} \pm 30^{\circ} \mathrm{C}$ from few minutes to several hours. CR-39 is the most sensitive and the highest resolution NTD. It is sensitive to a wide range of charges down to $Z=1-6 \mathrm{e}$ (depending on the $Z / \beta$ of the incident particle. Its charge resolution of $0.05 \mathrm{e}$ for precisely designed exposure and etching experiments. CR-39 was used to search for exotic particles, like Magnetic Monopoles and Strange Quark Matter (SQM) to study cosmic ray composition and for environmental studies [26].

\section{Experimental Methodology}

\subsection{Target-Detector Assemblies and Exposures}

Nuclear track detectors can be employed for the measurement of fragmentation cross sections of relativistic projectiles using appropriate target-stack designs. We have employed CR-39 detectors (prepared by Intercast Europe Company of Parma, Italy) for fragmentation studies of relativistic projectiles on various targets. The method of these experiments is the measurement of the beam incident on the target and after the target where are present the survived beam and the fragments or seconddary particles produced in the interaction of the incident beam with the target.

The design of target-detector assemblies and exposure geometry are shown in Figure 1. Several such stacks of CR-39 detectors and targets were exposed to $158 \mathrm{~A} \mathrm{GeV}$ ${ }^{207} \mathrm{~Pb}$ beams at the CERN SPS facility. The target thicknesses were chosen in such a way that multiple interactions were negligible, but still appropriate to produce sufficient fragments. The CR-39 detector plates were $11.5 \times 11.5 \mathrm{~cm}^{2}$ in size and $600 \mu \mathrm{m}$ in thickness. CR-39 detectors upstream of the targets were used for the determination of the total number of incident $\mathrm{Pb}$ ions. The density of lead beam ions was around 1500 ions $/ \mathrm{cm}^{2}$. The beam then passed through a target of thickness typically half of the mean free path of the $\mathrm{Pb}$ ions in the

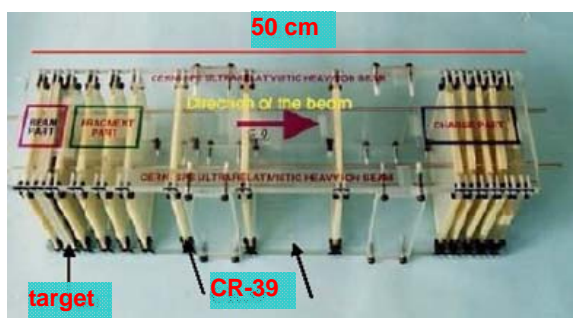

(a)

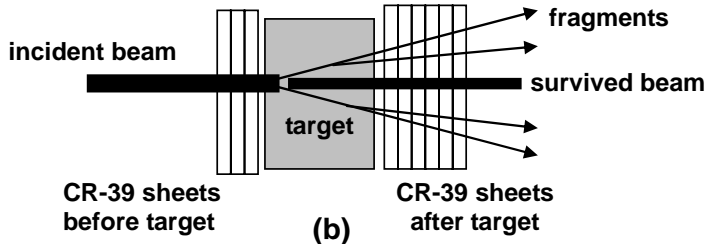

Figure 1. Schematic showing (a) Stack of NTDs and (b) exposure geometry.

given target. CR-39 foils downstream of the target recorded both the survived $\mathrm{Pb}$ ions and their fragments produced in targets. NTD based experiments are very simple compared to those which employ multi wire proportional chambers (MWPCs), ionization chambers (ICs) and Cherenkov detectors (CDs) $[27,28]$. The later experiments necessitate an online data acquisition whereas NTDs provide the possibility of offline data acquisition and are attractive for research groups which do not have direct access to high energy accelerator facilities.

\subsection{Chemical Etching}

Chemical etching is an essential step for NTD experiments. Etching amplifies the nanometer diameter particle trails in NTDs to microscopic dimensions appropriate for observations with optical microscopes. Nuclear track etching involves a number of factors without precisely quantized control. Most important factors are concentrations of active etching species or molecules in etchant, temperature control and stability. The variation in etching behavior of the detector material causes uncertainty in experimental etching results. The variation in the etching temperatures was not more than $\pm 1^{\circ} \mathrm{C}$. A standardized process was used during etching of exposed CR-39 detectors. An efficient and stable level of stirring was maintained. Concentration of etchant was kept effectively constant under specific etching conditions by minimizing the water evaporation from the etching solution. Exposed CR-39 detectors were etched in $4 \mathrm{~N} \mathrm{KOH}$ water solution at a temperature of $45^{\circ} \mathrm{C}$ for $72 \mathrm{~h}$. An average thickness of about $8 \mu \mathrm{m}$ was removed from both sides of the detector with an average bulk etch rate of $0.112 \pm 0.021 \mu \mathrm{m} / \mathrm{h}$. The refractive index of the CR-39 was experimentally determined as 1.561 which was later used for the determination of the total height of the etched cones. 


\subsection{Track Measurements}

Measurement of track parameters is an extremely slow process if it is done manually. All the track measurements at the PINSTECH laboratory were done manually. Manual measurements are very slow compared with automatic scanning systems, but provide visual insights of track forming particles. Discrimination between real events and background or erroneous events is more trustworthy in manual measurements compared to the discrimination made by an automatic nuclear track scanning. The measurement of cone heights of tracks in three CR-39 foils for the case of each target used, one set of foils for each target used, one set of foils before every target and two after, were measured with an optical microscope manually (more than 6000 etched cones were measured in each foil). It was observed that the cone length increased with increasing ion charges. Measurements of nuclear fragmentation were made with observations of change in cone heights. Zeiss optical microscope with a magnification of $40 \times$ was used for these measurements. Cone heights of $\mathrm{Pb}$ projectiles and fragments were measured by the top view using the depth measuring system coupled with the microscope. Difference of readings on depth measuring instrument, one when track diameter at the detector surface is focused and other when endpoint of etchable range is focused in the microscope, gives the cone height of a track. Track length measurement resolution or least count of the optical microscope used was $\pm 0.5 \mu \mathrm{m}$.

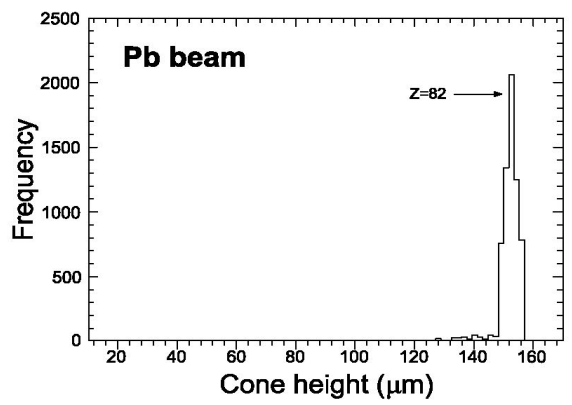

(a)

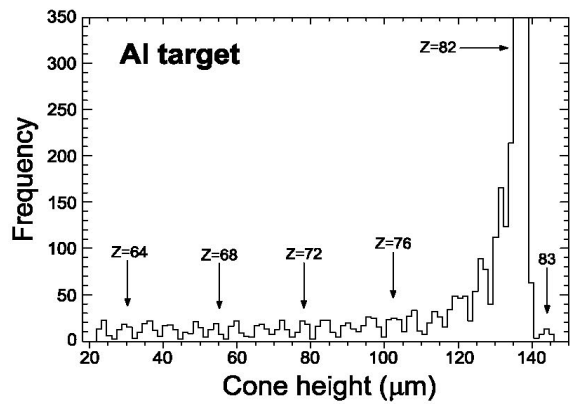

(c)

\section{Fragmentation Results}

\subsection{Detector Observations}

Figure 2 shows the etched cone distribution for tracks of lead projectiles (a), and fragments produced in the interaction of $\mathrm{Pb}$ ions on stationary CR-39 (b), $\mathrm{Al}$ (c) and $\mathrm{Cu}$ (d) targets, observed with CR-39 detector. In each plot, each peak can be designated with a charge $Z$ as most of the projectile energy is deposited in a solid (CR-39 here) through Coulomb interaction. In all the three cases of $\mathrm{Pb}$ projectile fragmentation in different targets (b, c and d), fragments down to $Z$ value of 63 are observable in CR-39. Counts in each peak are a measure of production cross section of the corresponding fragment. So, each peak represents production yield of fragments with a specific $Z$. Well-defined and separated peaks mean precise measurements. It is worth mentioning that detector, beam and etching conditions were same for all plots, but range of etched cone height is different for the case of $\mathrm{Al}(\mathrm{C})$ from other three cases. Major differences are difference in brands of $\mathrm{KOH}$ used and different chemical etching equipment. Major differences in etching equipment include difference in size and different magnitudes of stirring during etching.

\subsection{Calibrations}

Calibration here refers to the standardization of the particle detection response. Calibration is normally a curve or a relationship between two parameters, one of which is

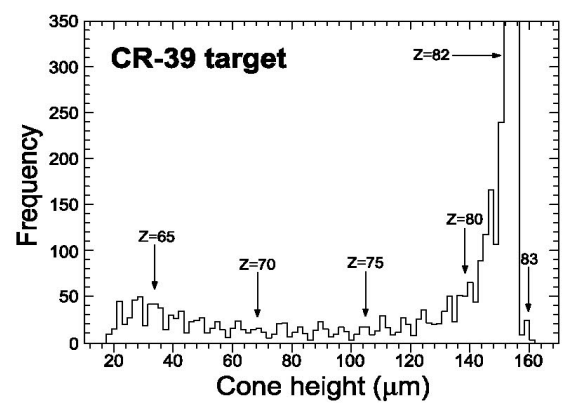

(b)

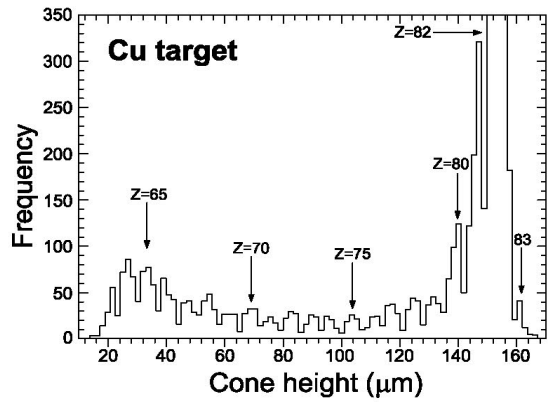

(d)

Figure 2. Etched cone distribution for tracks of lead projectiles (a), and their fragments produced in CR-39 (b), Al (c) and Cu (d) targets, observed with CR-39 detector. 
based on a detector observation or measurement and other related to a physical property of the incident particle. Such a measurement curve or relationship represents the detection response of a detector and can be employed to characterize unknown particles. In the present case, the etched cone distribution in Figure 2 was used to calibrate the CR-39 plastic track detector. It is an important step which standardizes response of the detector under a specific set of conditions for the experiment. Etching conditions and precision and repeatability of etching procedures have a strong influence on validity of the calibration.

Figure 3 shows the quantitative relationship between measured etched cone height and correlated fragment charge. A linear relationship is observed between cone height and corresponding fragment charge $Z$. Equation of the fitted line is shown in the plot along with regression coefficient and standard deviation, demonstrating excellent fit. Figure 4 shows the reduced etch rate "p" vs "REL" value. The error bars include statistical and systematic uncertainties. The points are the experimental data and the solid line is the best fit to the data points. The fitted equation is valid only in the REL range 3700 to $7100 \mathrm{MeV} \cdot \mathrm{cm}^{2} / \mathrm{gm}$. This plot can be used to identify unknown particles registered in CR-39 using same etching conditions by determining REL value of the unknown particle corresponding to the measured reduced etch rate "p".

\subsection{Charge Resolution}

The charge resolution, $\Delta Z$, of CR-39 in the charge region $63 \leq Z \leq 83$ is shown in Figure 5. Grey bar in the plot shows the average value of charge resolution and

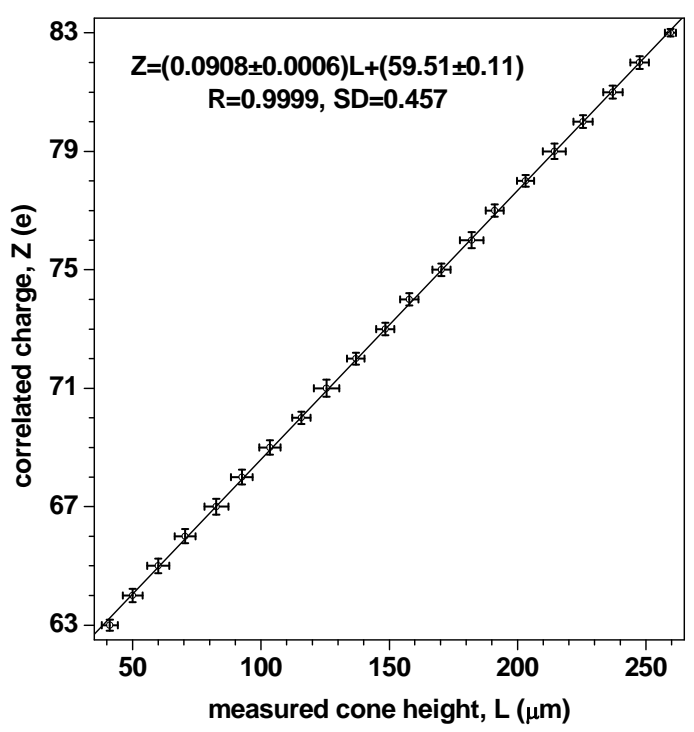

Figure 3. Quantitative relationship between measured etched cone height and fragment charge.

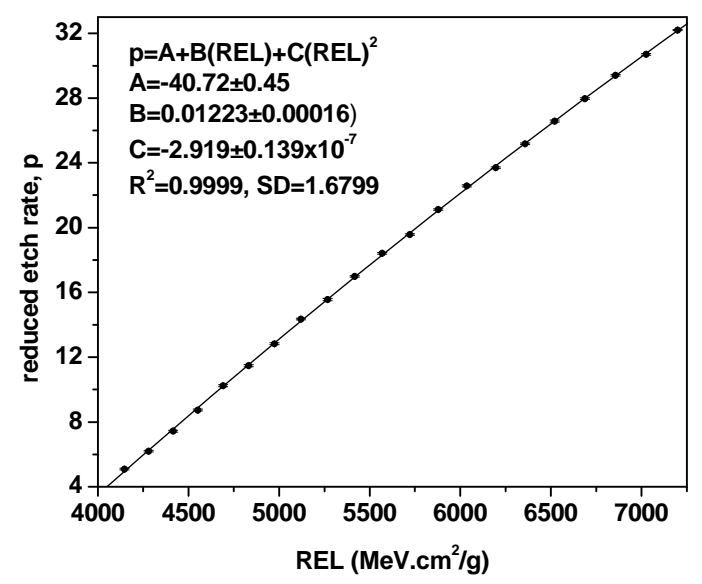

Figure 4. Reduced etch rate "p" vs "REL".

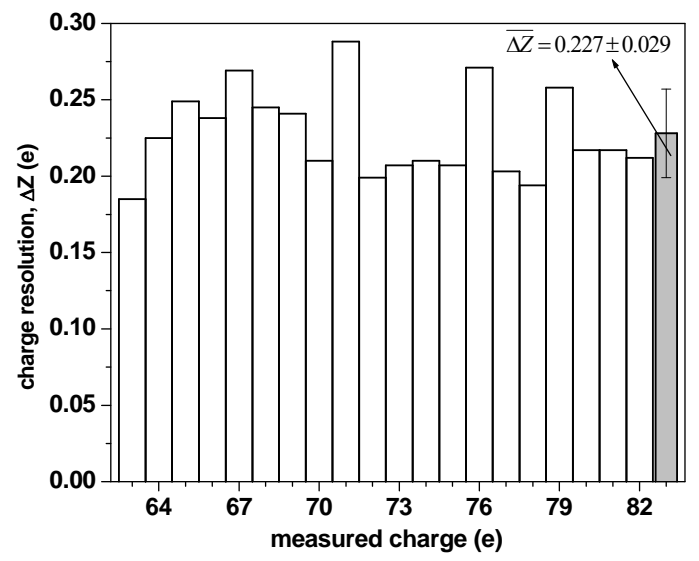

Figure 5. Charge resolution of CR-39 in the charge region $63 \leq \mathrm{Z} \leq 82$.

error bar on it is the standard deviation. The value of $\Delta Z$ decreases slowly with decreasing charge $Z$. The parameter $\Delta Z$ depends on a number of factors. Energy deposition rate $\mathrm{d} E / \mathrm{d} x$ or REL, damage responsiveness of the detector to $\mathrm{d} E / \mathrm{d} x$ [15], chemical etching conditions and procedure, and track measurement least count are important systematic factors determining $\Delta Z$. Statistical factors degrade the charge resolution and have a number of sources. Some of them are global like event number statistics and other local like local statistical variations in etching parameters.

Figure 6 shows the charge resolving power $(\eta)$ of CR39 detector. Charge resolving power is a measure of the ability of a detector to observe a charge resolved. It is reciprocal of the charge resolution $\Delta Z$ for a specific charge $Z$ and is defined as,

$$
\begin{gathered}
\eta=\frac{1}{\Delta Z / Z}, \\
\eta=\frac{Z}{\Delta Z} .
\end{gathered}
$$




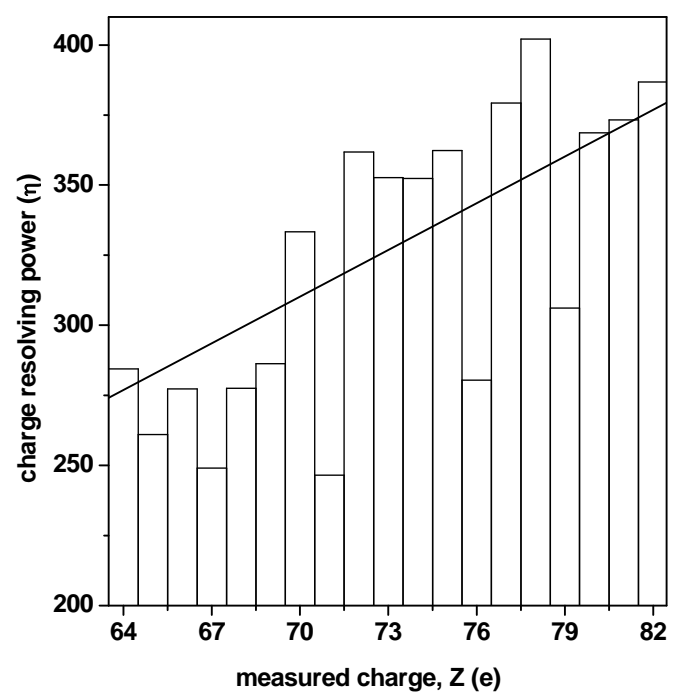

Figure 6. Decreasing resolving power of CR-39 with charge Z.

Charge resolving power CR-39 decreases with charge. Higher charge means higher energy deposition rate $\mathrm{d} E / \mathrm{d} x$ or REL which reflects into enhanced resolving power $\eta$. For the present reaction and etching conditions cannot detect or resolve charge below 63 .

\subsection{Fragmentation Cross Sections}

Figure 7 shows our experimentally measured partial charge-changing cross sections of $158 \mathrm{~A} \mathrm{GeV} \mathrm{Pb}$ on $\mathrm{Cu}$ target, as an example. For comparison, cross section measurements of the same projectiles with the same energy on the same target measured by Schiedenberger et al. [3] using an active detector, multi sampling ion chamber (MUSIC). The inset plot shows the comparison of measurement errors. Detection range of the MUSIC detector $(Z=60-82)$ is wide than that of CR-39 in the present case $(Z=63-82)$. But, in general our measurement error is comparable to that of MUSIC and are slightly better for $Z=76-82$. Our measurements do not allow us to say anything directly about mass changing resolution of fragments as detectors used were only sensitive to charge of fragments and not to their mass.

\section{Comparative Merits and Demerits}

\subsection{Merits}

\subsubsection{General}

The NTDs are single particle detectors. Every individual particle is recorded as a separate event in a NTD for a certain dose or fluence of particles for which events do not overlap. Using the traditional NTD methodology with chemical etching and optical microscopy, a dose of particles up to $10^{5} \mathrm{~cm}^{-2}$ can be measured. The present use of AFM can extend the observable dose limit up to $10^{8}$

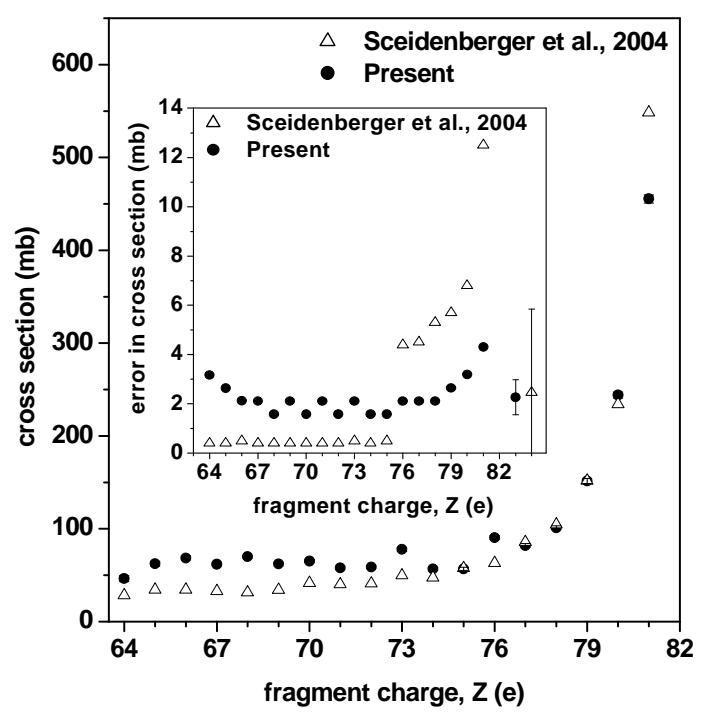

Figure 7. Present CR-39 158 A GeV Pb projectile fragmentation cross section measurements in comparison with corresponding online measurements by Schiedenberger et al. [3]. Inset plot compares the measurement errors.

$\mathrm{cm}^{2}$. Important useful features of NTD include small and simple experimental setups, integrated exposure response, robust nature, no use of sophisticated electronics and low capital and operational costs. A method, called fission track dating (FTD), is based on nuclear tracks and is employed to unfold the thermal history of rocks [31].

\subsubsection{Specific to Fragmentation}

NTDs have several advantages over other techniques with the consideration specific to nuclear fragmentation or reaction studies. A NTD based experiment is shorter $(\sim 50 \mathrm{~cm})$ than others: a set up with the MUSIC detector has a length of $2 \mathrm{~m}$ [3]. So, a NTD experimental setup can easily be coupled to the beam exit point at a high energy accelerator facility. No triggering system for data acquisition not needed in NTD experiments while it is required in electronic experimental setups. Normally, one uses several exposure spills in the case of a NTD target-detector assembly. One spill can be studied as a test case. The procedure of chemical etching can be optimized offline. Incident beam particles and fragments or other particles produced in the reaction are permanently recorded in a NTD without any sophisticated technology.

\subsection{Demerits}

\subsubsection{General}

Tedious efforts are required to achieve high charge and energy resolution. Most requirements in this regard are related to precision and controls in detector properties, especially uniformity, precautions in exposures and chemical etching. Information about the mass of the incident particles can be deduced with a considerable error. 
It lacks real time information about incident particles. No unified and clear physical and mathematical picture of nuclear track formation is available. Nuclear track etching is an essential but still a wild procedure due to the involvement of a number of parameters without quantitative microscopic control. Even microscopic picture of nuclear track etching is well understood. Etch induction time is an example of lacks of understanding. Concentration of active etching species, control on etchant temperature, and variation in etching behaviour of the detector material are major causes of uncertainty and wildness of chemical etching.

\subsubsection{Specific to Fragmentation}

Apart from NTDs, other detectors in use for high energy nuclear fragmentation are ionization chambers (ICs), wire chambers (WCs), semiconductor detectors (SeDs), scintillation detectors $(\mathrm{ScDs})$ and Cherenkov counters (CCs) $[27,28]$. They are online detectors and have a certain superior characteristics. For example, MUSIC is a charge sensitive detector having a smaller number of atoms $/ \mathrm{cm}^{2}$ compared with solid NTDs $[29,30]$. So, in MUSIC there are less secondary hadronic interactions. Much better statistics can be achieved using electronic detectors. Track statistics is limited in NTDs by a certain dose as they record all the particles. So, a fluence of $10^{2}$ $10^{4} / \mathrm{cm}^{2}$ is a good achievable statistics. It may be noted that achievable statistics depend on diameters of particle tracks and high statistics increase error is statistics due to the overlapping of tracks.

\subsection{Nuclear Fragmentation versus Fullerene Fragmentation and Quark Gluon Plasma}

There are two possibilities for the fragmentation of highly excited nuclei. First is the case when fragmentation products depend on magnitude of the excitation energy induced by the interacting projectile whereas in the second case they depend on magnitude and nature of excitation. The second possibility has some relevance with the case of fragmentation of fullerenes. Fullerene is a cluster of atoms forming a structure with narrow cages whereas nucleus is a cluster of nucleons moving inside in a complex manner. Despite bonding nature of constituents of nucleus and fullerene differs significantly, fragmentation of these two objects may have commonalities. It is difficult to apprehend the nature of excitation of a highly excited nucleus due to involvement of a large number of degrees of freedom some of which still need to be further understood. [32,33]. Another interesting outlook realized in this study is the formation of shock wave in relativistic nuclear collision similar to that in fusion plasma in which nuclei collide to hammer each other producing a shocked high density nuclear material which may undergo some oscillations [34] before its fragmentation in somewhat the same way as fullerene under ion bombardment. The shock approach is valid for both central and peripheral interactions due to their penetration into each other, which was initially was proposed by Siemiarczuk and Zielinski in fragmentation of Xe nuclei induced by negative pions [35]. The oscillations in the shock are damped due to energy release through evaporation of particles and/or fragmentation [36]. Formation of shock is more probable in central collisions case where the nuclear material may undergo through a stage similar to plasma dust crystal [37,38] leading the multi-fragmentation. Due to variance of density of in the collided nuclear material, the formation of dispersive wave can form density varying rings, like Saturn's B ring. Scientifically-based technical chronological details of these rings have been provided by Massod et al. [39] taking the support of the Hubble Space telescope observations of the "spokes".

\section{Co-Use of NTD with Other Techniques}

In physics experiments, complete identification of a particle means information about its energy, charge and mass. In most experiments, limited measurements of particle parameters are made and more information is made available by correlating detector observations with the theory and already available information about particles or reactions involved. Every technique has specific merits and demerits, so use of more than one technique can enhance the information about the nuclear reaction and its precision. NTDs remained slightly underdeveloped owing to their passive nature. In a recent study, it was investigated how precision and reliability can be achieved in experiments using NTDs [40]. The NTD allow tracking of particles with measurement of their energy and charge. So, despite their passive nature, NTDs could supplement electronic measurements.

Figure 8 shows a schematic proposing the co-use of NTDs with online electronic techniques. Such a design

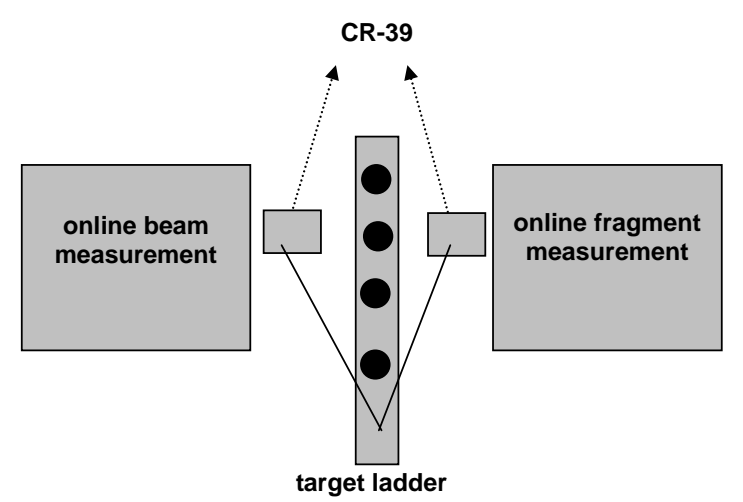

Figure 8. Schematic proposing the co-use of NTD with online techniques for the measurement of fragmentation cross sections. 
could be useful regarding precision, reliability and widening of scope of the experiment. In the proposed design, the use of three fold ladder is suggested. The central arm will serve as a target as in other experiments with active detectors. The other 2 arms will hold CR-39 samples for a defined time, one upstream and other downstream of the target, respectively for the measurement of projectiles and their fragments. Co-use of active and passive detectors could add confidence and reliability. NTDs are limited to $10^{4}-10^{6}$ projectiles $/ \mathrm{cm}^{2}$. When the defined dose of projectiles is reached, the CR-39 detectors could be removed leaving online electronic experiments in place. Such experiments could help involving researchers from developing countries where lengthy part of track measurements in NTDS is possible.

In some large experiments, this combination was made: for example the MACRO (monopole astrophysics cosmic ray observatory) [41,42] experiment in which NTDs were used as a subdetector. The NTD subdetector was used in the middle and in two sides of its lower structure [43]. NTDs are suitable for cosmic ray studies including the searches for exotic particles. They have been employed for such measurements with good precision [44-47]. One half of 32 searches for magnetic monopoles (MMs) were performed with NTDs. Monopole and Exotics Detector at the LHC (MoEDAL) aims to search for MMs at CERN LHC [48]. Co-use of NTDs with other techniques [49-53] would enhance their use for cosmic rays and other exotic particle searches for which they are most suitable due to experimental simplicity, light weight and low cost.

Figure 9 is an artistic portraying of the finding reported in paper and related. It also includes segments on the field of radiation detection \& measurements and the nuclear safety which are related to the subject of this article.

\section{Conclusion}

Selected results of experiments, carried out over many years with the main aim of precise measurements of relativistic nuclear projectile fragmentation cross sections and related processes using NTDs, were discussed and we cover some major aspects of nuclear track detection technique. The experimental precautions described here for exposure, chemical etching and track measurements are applicable to experiments in a laboratory with facilities considered essential for NTDs. Possible experimental refinements in nuclear track etching are discussed. Fragmentation results obtained with CR-39 detectors have been compared with competing active detectors. Issues of charge resolution and charge resolving power of CR-39 were discussed. The results discussed may be useful for the community involved with applications of nuclear instruments and methods, especially in studies of nuclear reactions and cosmic rays.

\section{Acknowledgements}

We acknowledge the cooperation of the staff of the CERN SPS for exposures. Thanks are also for the technical help by Mr. Bashir, Mr. Amjad, Mr. Ashiq, Mr. Saeed and Mr. Rizwan at PINSTECH. Remembering back my (MAR) Ph.D. (2001-2005) period at Center for Ion Beam Applications (CIBA), Physics Department, Faculty of Science, the National University of Singapore (NUS), the kind help of CIBA members (Thomas, Mark, Frank, Jeroen, Andrew, Chamika, Minqin, Taw Kuei, Piravi, Ce-Belle, Choo Theam, Armin, Yan John, Xiong Aky, Sara, Chen Kyle, Mallikarjuna Rao, Vanga, Liang Haidong, Dang Zhiya, Song Jiao, Wang Yinghui, Liu Nan, Liu Fan, Wu Feng, Yang Chenyuen, Yao Yong, Prashant, Chan Fun, Homhuan Susan, Hoi Kit, Anna Rodrigues, Chiam Sheryi, Reshmi Rajendarn, Raj Kumar, Liu Tiancai, Brandon Ho, Zhang Fang, Daniel Parker, Martin Wei, Kambiz Mahabadi, Debbie Seng, Wang Liping, Tze Chien, Chan Fun, Ow Isaac, Shripad, Anna Rodrigues, Chiam Sheryi, Liu Tiancai, Mangaiyarkarasi, Huang Long, Zheng Zhong; Hai Long, Soma Rao, S.M. Tang, Markus Zmeck (Lehrstuhl für Elektronik, Fachbereich Elektrotechnik, Universität Wuppertal, Fuhlrottstr. 10, Wuppertal D-42097, Germany), Changyi Yang, Patricia Thong, Jose Sanchez, Ivo Orlic, S.J. Zhou) is gratefully acknowledged with special thanks to my supervisors A/Prof. Thomas Osipowicz and Prof. Mark Breese and CIBA Director of that time Prof. Frank Watt for their guidance. Many thanks to engineer Theam Choo for his technical help during experiments. The guidance of and discussion with Leszek Wielunski, Andrew Bettiol, Jeroen van Kan, Ms Minqin Ren, Shao Peige, Istvan and Muhammad on several occasions is thankfully acknowledged. The help of Huang Long and Markus Zmeck during experiments is also appreciated. Contributions from our collaborators, Dr. Ian Watson at Institute of Photonics, University of Strathclyde, UK. Prof S.J. Chua, Dr. Anthony Choi, Dr. Chen Peng at Center for Optoelectronics, Faculty of Engineering, NUS. Mr Y.Y. Liu and Assoc Prof Thong Leong at Centre for Integrated Circuit Failure Analysis and Reliability, Faculty of Engineering, NUS. Assoc Prof Andrew Wee at Surface Science Laboratory, Faculty of Science, NUS. Assoc Prof Shen Xiang and Dr. Wang Sun at Department of Physics, Faculty of Science, NUS is gratefully acknowledged. Very thankful to Peter Smulders at University of Groningen, Netherlands, who computer code FLUX used for ion channelling simulations presented in the thesis; James Pinfold, University of Alberta, spokesperson for the MoEDAL (an LHC experiment at CERN, Switzer- 


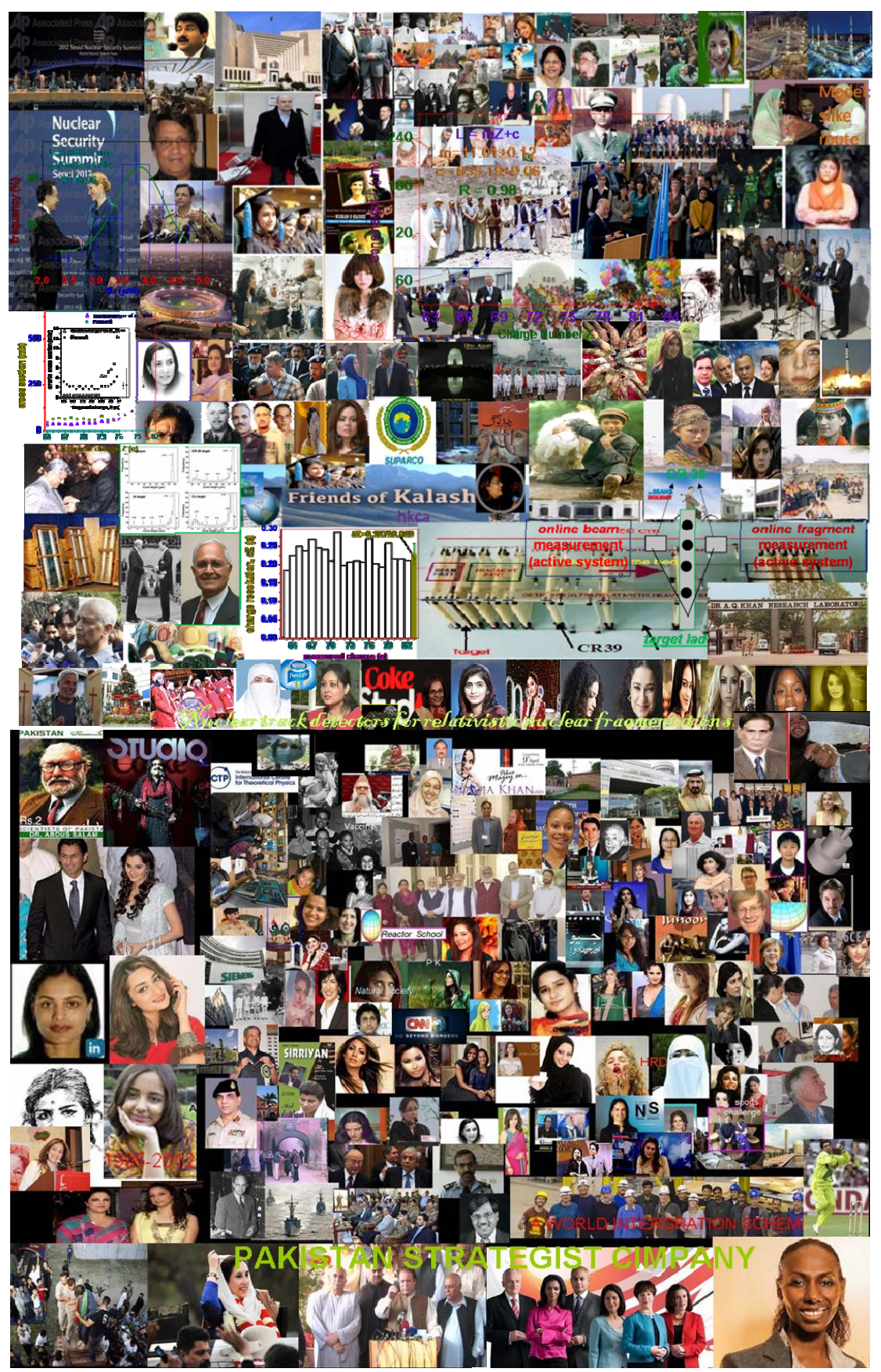

Figure 9. Artistic portraying of the finding reported in paper and related. It also includes segments on the field of radiation detection \& measurements and the nuclear safety which are related to the subject of this article. In bottom half, global integration scheme, considered the most suitable for the nuclear safety/security, is portrayed. It is an artist-eye view for the broader scope of readers of the scientific literature. Scientific data/results are original or adopted from the scientific literature (most cited in this article in references list). Related pictures are from several digital online/passive resources/search engines, including IAEA Websites, CNN (US), BBC (Britain), ARY TV, Sama TV, Pakistan News Press, PTV, Din News TV, Koh-e-Noor TV, WE TV, Wikipedia, Google, SPD, PAEC, PINSTECH, PIMS, MH (Pakistan), Centre for Ion Beam Applications (CIBA), National University of Singapore (Singapore), University of Manchester, NDA (UK), International Islamic University (IIU), Islamabad (Pakistan), KINPOE, Karachi (Pakistan), NUST, Air University, Bahria University, PAEC Hospital Library, MISI (CDA), PAEC Head Quarters Centre for the Nuclear Safety, PIMS, Islamabad (Pakistan), Military College Jhelum, Jhelum (Pakistan), AS-ICTP, Trieste (Italy), Instituto Tecnológico e Nuclear, Estrada Nacional 10, $2686-953$ Sacavém, CICECO, Departamento de Física, Universidade de Aveiro, 3810-193 Aveiro, Portugal, University of Strathclyde, Glasgow G4 0NW, UK, Fermi Lab, NASA, Los Alamos, WM2013 (US), GSI (Germany), Fukushima related Japanese/IAEA Websites and several others. 
land) collaboration.

Discussions with following ones are gratefully acknowledged: Eugene (Gene) A. Rosa (Boeing Professor of Environmental Sociology and Professor of Fine Arts, Washington State University, USA; Mr. John Mathieson, Nuclear Decommissioning Authority, Curie Avenue, Harwell, Didcot, Oxon, UK; Dr. Khawja Dr. Noor Muhammad Butt, Nanoscience and Technology, Preston University, Islamabad, Pakistan; Dr. Sabiha Mansoor, Ms. Rodaba Hamid (LCWU, Lahore, Pakistan); Dr. Maria Sultan, Mr. Nick Robson, SASSI, London, Nancy Kalish, Professor Emeritus of Psychology, California State University, Sacramento, USA; Dr. Karl-Heinz. Schmidt, GSI, Germany; Dr. Dietrich Hermsdorf, Dresden, Germany; Dr. Ghulam Rasul (Pak. Meteorological Dept., Islamabad) in Keynote Address on Impacts of the Global Warming and the Climate Change, at Dr. Dr.I.H. Usmani Auditorium, PINSTECH, 17-19 Sep, 2012, organized by Isotopes Applications Division, Directorate of Technology, PINSTECH, Islamabad; Dr. Laureline Bourcier, Joint Research Center, European Commission, B-2440 Geel, Belgium; Olivier Masson, Institut de Radioprotection et de Sûreté Nucléaire (IRSN), Cadarache, BP 3, 13115 saint Paul lez Durance, France; Ms. Selly Soetarso, WM Symposia2013; Brg. Ghazanfar Dar, Mirza Khurshid Alam (Nilore); Several people at Darussalam and Masjid Mujahideen (Singapore); Mr. Shaukat Hassan, Qasim Ali Shah, D.G. Khan; T.D. Märk, Department of Plasma Physics, Comenius University, SK84248 Bratislava, Slovak Republic.Institut fur Ionenphysik, Leopold Franzens Universitat, Technikerstr. 25, A-6020, Innsbruck, Austria; Seminars/lectures at University of Manchester (UK): Sara Whitehouse, John Hubbuck, John Keating, Karl Coleman (University of Durham, UK), Louise Walker, Muhammad Mohiuddin, Krzysztof Goscinski; Dr. Inam-ur-Rahman, a Nuclear Engineering teacher who raised an Engineering University (PIEAS, Islamabad) with specific central scopes of Nuclear Engineering, Systems Engineering and Nuclear Medicine and other Science and Engineering Management disciplines. A beautiful, innocent and a natural folk Arabic styled Pakistani singer Reshman who was born in Rajisthan in 1947 and moved to the nucleating Pakistan. Her true talent remained candid and could not be surfaced. Despite not having professional education, she famed very high in the world of the music, especially in the folk, exposing or melonizing the occupied signatures of the remote culture; Brig (R) Muhammad Afzal Malik, Military College Jhelum, Capt. Umair Nazir (Cadet College Hassan Abdal) and Nilore, Islamabad), Anjum Qureshi, Shahzeb Pervaiz (NUS, Singapore); Lecturers at IAEA-ICTP Radiation Damage Workshop, AS-ICTP, Trieste, Italy: DYTLEWSKI Nikolai (Australia), INOZEMTSEV Victor (Russian Federation), SALAME
Petra (Lebanon), SCANDOLO Sandro (Italy) ZEMAN Andrej (Slovak Republic), COPPOLA Roberto (Italy), FREYSS Michel (France), KOHANOFF Jorge Jose (United Kingdom) KURIPLACH Jan (Czech Republic) LAZAREV Nikolay (Ukraine), MALERBA Lorenzo (Italy), PERLADO Jose Manuel (Spain), ROBERTSON Christian (France), RYAZANOV Alexander (Russian Federation), SAMARAS Maria (Greece), VICTORIA Maximo (Argentina), WIESENACK Wolfgang (Germany).

\section{REFERENCES}

[1] M. Zmeck, J. Phang, A. Bettiol, T. Osipowicz, F. Watt, L. Balk, F.-J. Niedernostheide, H.-J. Schulze, E. Falck and R. Barthelmess, "Analysis of High-Power Devices Using Proton Beam Induced Charge Microscopy," Microelectronics Reliability, Vol. 41, No. 9-10, 2001, pp. 15191524. doi:10.1016/S0026-2714(01)00159-7

[2] J. W. Wilson, J. L. Shinn, L. W. Townsend, R. K. Tripathi, F. F. Badavi and S. Y. Chun, "NUCFRG2: A Semiempirical Nuclear Fragmentation Model," Nuclear Instruments and Methods in Physics Research Section B: Beam Interactions with Materials and Atoms, Vol. 94, No. 1-2, 1994, pp. 95-102. doi:10.1016/0168-583X(94)95662-6

[3] C. Scheidenberger, et al., "Charge-Changing Interactions of Ultrarelativistic Pb Nuclei," Physical Review C, Vol. 70, No. 1, 2004, Article ID: 014902. doi:10.1103/PhysRevC.70.014902

[4] L. Y. Geer, J. Klarmann, B. S. Nilsen, C. J. Waddington, W. R. Binns, J. R. Cummings and T. L. Garrard, "Charge-Changing Fragmentation of $10.6 \mathrm{GeV} /$ Nucleon ${ }^{197}$ Au Nuclei," Physical Review C, Vol. 52, No. 1, 1995, pp. 334-335. doi:10.1103/PhysRevC.52.334

[5] W. R. Weber, J. C. Kish and D. A. Schrier, "Total Charge and Mass Changing Cross Sections of relativistic nuclei in Hydrogen, Helium, and Carbon Targets," Physical Review C, Vol. 41, No. 2, 1990, pp. 520-532. doi:10.1103/PhysRevC.41.520

[6] W. R. Weber, J. C. Kish and D. A. Schrier, "Individual Charge Changing Fragmentation Cross Sections of Relativistic Nuclei in Hydrogen, Helium, and Carbon Targets," Physical Review C, Vol. 41, No. 2, 1990, pp. 533546. doi:10.1103/PhysRevC.41.533

[7] C. Zeitlin, S. Guetersloh, L. Heilbronn, J. Miller, A. Fukumura, Y. Iwata, T. Murakami, L. Sihver and D. Mancusi, "Fragmentation Cross Sections of MediumEnergy ${ }^{35} \mathrm{Cl},{ }^{40} \mathrm{Ar}$, and ${ }^{48} \mathrm{Ti}$ Beams on Elemental Targets," Physical Review C, Vol. 77, No. 3, 2008, Article ID: 034605. doi:10.1103/PhysRevC.77.034605

[8] K.-H. Schmidt, A. Kelić and M. V. Ricciardi, "Experimental Evidence for the Separability of Compound-Nucleus and Fragment Properties in Fission," Europhysics Letters, Vol. 83, No. 3, 2008, Article ID: 32001. doi:10.1209/0295-5075/83/32001

[9] G. Giacomelli, et al., "Extended Calibration of a CR39 Nuclear Track Detector with 158 A GeV. ${ }^{207} \mathrm{~Pb}$ Ions," 
Nuclear Instruments and Methods in Physics Research Section A: Accelerators, Spectrometers, Detectors and Associated Equipment, Vol. 411, No. 1, 1998, pp. 41-45. doi:10.1016/S0168-9002(98)00270-8

[10] S. Cecchini, et al., "Fragmentation Cross Sections of $\mathrm{Fe}^{26+}, \mathrm{Si}^{14+}$ and $\mathrm{C}^{6+}$ Ions of $0.3-10 \mathrm{~A} \mathrm{GeV}$ on Polyethylene, CR39 and Aluminum Targets," Nuclear Physics A, Vol. 807, No. 3-4, 2008, pp. 206-213. doi:10.1016/j.nuclphysa.2008.03.017

[11] S. Manzoor, et al., "Nuclear Track Detectors for Environmental Studies and Radiation Monitoring," Nuclear Physics B-Proceedings Supplements, Vol. 172, 2007, pp. 92-96. doi:10.1016/j.nuclphysbps.2007.07.017

[12] S. Balestra, et al., "Bulk Etch Rate Measurements and Calibrations of Plastic Nuclear Track Detectors," Nuclear Instruments and Methods in Physics Research Section B: Beam Interactions with Materials and Atoms, Vol. 254, No. 2, 2007, pp. 254-258. doi:10.1016/j.nimb.2006.11.056

[13] W. Heinrich, et al., "High Energy Heavy Ion Interactions Studied with SSNTDs," Radiation Measurements, Vol. 25, No. 1-4, 1995, pp. 203-218. doi:10.1016/1350-4487(95)00075-P

[14] S. Kodaira, N. Yasuda, H. Kawashima, M. Kurano, N. Hasebe, T. Doke, S. Ota, T. Tsuruta, H. Hasegawa, S. Sakai, T. Nishi and K. Ogura, "Characteristics of the Copolymerized CR-39/DAP Track Detector for the Observation of Ultra Heavy Nuclei In Galactic Cosmic Rays," Radiation Measurements, Vol. 44, No. 9-10, 2009, pp. 775-778. doi:10.1016/j.radmeas.2009.10.050

[15] P. B. Price and Y. D. He, "Behavior of Nuclear Projectile Fragments Produced in Collisions of $14.5 \mathrm{~A} \mathrm{GeV}{ }^{28} \mathrm{Si}$ with $\mathrm{Pb}$ and $\mathrm{Cu}$ Targets," Physical Review C, Vol. 43, No. 2, 1991, pp. 835-848. doi:10.1103/PhysRevC.43.835

[16] M. Giacomelli, L. Sihver, J. Skvarč, N. Yasuda and R. Ilić, "Projectilelike Fragment Emission Angles in Fragmentation Reactions of Light Heavy Ionsin the Energy Region <200 MeV/Nucleon: Modeling and Simulations," Physical Review C, Vol. 69, No. 6, 2004, Article ID: 064601. doi:10.1103/PhysRevC.69.064601

[17] A. N. Golovchenko, J. Skvarc, R. Ilić, L. Sihver, V. P. Bamblevski, S. P. Tretyakova, D. Schardt, R. K. Tripathi, J. W. Wilson and R. Bimbot, "Fragmentation of 200 and $244 \mathrm{MeV} / \mathrm{u}$ Carbon Beams in Thick Tissue-Like Absorbers," Nuclear Instruments and Methods in Physics Research Section B: Beam Interactions with Materials and Atoms, Vol. 159, No. 4, 1999, pp. 233-240. doi:10.1016/S0168-583X(99)00546-7

[18] A. N. Golovchenko, L. Sihver, S. Ota, J. Skvarč, N. Yasuda, S. Kodaira, G. N. Timoshenko and M. Giacomelli, "Fragmentation of $370 \mathrm{MeV} / \mathrm{n}^{20} \mathrm{Ne}$ and $470 \mathrm{MeV} / \mathrm{n}{ }^{24} \mathrm{Mg}$ in Light Targets," Radiation Measurements, Vol. 454, No. 7, 2010, pp. 856-860. doi:10.1016/j.radmeas.2010.03.006

[19] M. A. Rana, "Nuclear Tracks: Defect Structure of Latent Tracks, Chemical Etching and Future Directions," Nuclear Instruments and Methods in Physics Research Section B: Beam Interactions with Materials and Atoms, Vol. 268, No. 2, 2010, pp. 165-171. doi:10.1016/j.nimb.2009.10.171
[20] M. A. Rana, "On Problems in Trustworthy Predictions of Etched Track Parameters," Radiation Measurements, Vol. 43, No. 9-10, 2008, pp. 1546-1549. doi:10.1016/j.radmeas.2008.09.002

[21] M. A. Rana, E. U. Khan, M. I. Shahzad, S. Manzoor, G. Sher and I. E. Qureshi, "Characteristics of Antiproton Tracks in CR-39," Radiation Measurements, Vol. 42, No. 2, 2007, pp. 125-129. doi:10.1016/j.radmeas.2006.12.002

[22] S. Manzoor, I. E. Qureshi, M. A. Rana, M. I. Shahzad, G. Sher, M. Sajid, H. A. Khan, G. Giacomelli, M. Giorgini, G. Mandrioli, L. Patrizii, V. Popa, P. Serra and V. Togo, "Charge Identification in CR-39 Nuclear Track Detector Using Relativistic Lead Ion Fragmentation," Nuclear Instruments and Methods in Physics Research Section A: Accelerators, Spectrometers, Detectors and Associated Equipment, Vol. 453, No. 3, 2000, pp. 525-529. doi:10.1016/S0168-9002(00)00470-8

[23] I. E. Qureshi, M. I. Shahzad, M. T. Javed, S. Manzoor, G. Sher, F. Aleem and H. A. Khan, "Study of Projectile Fragmentation in the Reaction (158 A GeV) $\mathrm{Pb}+\mathrm{Pb}$ Using CR-39," Radiation Measurements, Vol. 40, No. 2-6, 2005, pp. 437-441. doi:10.1016/j.radmeas.2004.11.013

[24] M. A. Rana and S. Manzoor, "Examining the Fragmentation of 158 A GeV Lead Ions on Copper Target: ChargeChanging Cross Sections," Radiation Measurements, Vol. 43, No. 8, 2008, pp. 1383-1389. doi:10.1016/j.radmeas.2008.05.004

[25] G. Sher, M. I. Shahzad and M. Hussain, "Fragmentation of (158 A GeV) Pb Ions with Bi Target," Radiation Measurements, Vol. 42, No. 10, 2007, pp. 1692-1695. doi:10.1016/i.radmeas.2007.09.006

[26] M. Giorgini, "The Nuclear Track Detector CR39: Results from Different Experiments," Nuclear Physics B-Proceedings Supplements, Vol. 197, No. 1, 2009, pp. 66-69. doi:10.1016/j.nuclphysbps.2009.10.036

[27] C. Leroy, "Review of Radiation Detectors," AIP Conference Proceedings, Vol. 1204, 2009, p. 49.

[28] C. Leroy and P. G. Rancoita, "Principles of Radiation Interaction in Matter and Detection," World Scientific, Singapore, 2004.

[29] E. V. Benton and W. G. Nix, "The Restricted Energy Loss Criterion for Registration of Charged Particles in Plastics," Nuclear Instruments and Methods, Vol. 67, No. 2, 1969, pp. 343-347. doi:10.1016/0029-554X(69)90471-6

[30] S. Balestra, et al., "Bulk Etch Rate Measurements and Calibrations of Plastic Nuclear Track Detectors," Nuclear Instruments and Methods in Physics Research Section B: Beam Interactions with Materials and Atoms, Vol. 254, No. 2, 2007, pp. 254-258. doi:10.1016/j.nimb.2006.11.056

[31] N. U. Khattak, M. Akram, M. A. Khan and H. A. Khan, "Emplacement Time of the Loe-Shilman Carbonatite from NW Pakistan: Constraints from Fission-Track Dating," Radiation Measurements, Vol. 43, Suppl. 1, 2008, pp. S313-S318. doi:10.1016/j.radmeas.2008.03.038

[32] B. Jurado, C. Schmitt, K.-H. Schmidt, J. Benlliure, T. 
Enqvist, A. R. Junghans, A. Kelic and F. Rejmund, "Transient Effects in Fission from New Experimental Signatures," Physical Review Letters, Vol. 93, No. 7, 2004, Article ID: 072501. doi:10.1103/PhysRevLett.93.072501

[33] T. Kunert and R. Schmidt, "Excitation and Fragmentation Mechanisms in Ion-Fullerene Collisions," Physical Review Letters, Vol. 86, No. 23, 2001, pp. 5258-5261. doi:10.1103/PhysRevLett.86.5258

[34] A. D. Boozer, "Simulating a One-Dimensional Plasma," American Journal of Physics, Vol. 78, No. 6, 2010, pp. 580-584. doi:10.1119/1.3290442

[35] T. Siemiarczuk and P. Zielinski, "Fragmentation of Xenon Nuclei Induced by $9 \mathrm{GeV} / \mathrm{c}$ Negative Pions and the Possibility of Shock Wave Propagation in Nuclei," Physical Review C, Vol. 13, No. 6, 1976, pp. 2439-2443. doi:10.1103/PhysRevC.13.2439

[36] M. A. Rana, S. Manzoor and G. Sher, "Charge and Mass Changing Cross-Sections of $158 \mathrm{~A} \mathrm{GeV} \mathrm{Pb}$ Projectiles on $\mathrm{Al}, \mathrm{Cu}, \mathrm{Bi}$, and $\mathrm{Pb}$ Targets: Fragmentation Processes," International Journal of Modern Physics E, Vol. 19, No. 10, 2010, p. E19. doi:10.1142/S0218301310016466

[37] H. Thomas, G. E. Morfill, V. Demmel, J. Goree, B. Feuerbacher and D. Möhlmann, "Plasma Crystal: Coulomb Crystallization in a Dusty Plasma," Physical Review Letters, Vol. 73, 1994, pp. 652-656.

[38] C.-R. Du, K. R. Sütterlin, A. V. Ivlev, H. M. Thomas and G. E. Morfill, "Model Experiment for Studying Lane Formation in Binary Complex Plasmas," Europhysics Letters, Vol. 99, No. 4, 2012, Article ID: 45001.

[39] W. Masood, H. Rizvi, H. Hasnain and Q. Haque, "Rotation Induced Nonlinear Dispersive Dust Drift Waves Can be the Progenitors of Spokes," Physics of Plasmas, Vol. 19, No. 3, 2012, pp. 32112-32118. doi:10.1063/1.3688077

[40] M. A. Rana, "How to Achieve Precision and Reliability in Experiments Using Nuclear Track Detection Technique?" Nuclear Instruments and Methods in Physics Research Section A: Accelerators, Spectrometers, Detectors and Associated Equipment, Vol. 592, No. 3, 2008, pp. 354-360. doi:10.1016/j.nima.2008.04.025

[41] M. Ambrosio, et al., "The MACRO Detector at Gran Sasso," Nuclear Instruments and Methods in Physics Research Section A: Accelerators, Spectrometers, Detectors and Associated Equipment, Vol. 486, No. 3, 2002, pp. 663-707. doi:10.1016/S0168-9002(01)02169-6

[42] M. Ambrosio, et al., "Final Results of Magnetic Monopole Searches with the MACRO Experiment," The European Physical Journal C: Particles and Fields, Vol. 25, No. 4, 2002, pp. 511-522. doi:10.1140/epjc/s2002-01046-9

[43] M. Ambrosio, et al., "Magnetic Monopole Search with the MACRO Detector at Gran Sasso," Physics Letters B,
Vol. 406, No. 3, 1997, pp. 249-255. doi:10.1016/S0370-2693(97)00684-9

[44] P. B. Price, L. M. Cook and A. Markert, "Phosphate Glasses for Identification of Heavy Ions," Letters to $\mathrm{Na}$ ture, Vol. 325, 1987, pp. 137-138. doi: $10.1038 / 325137 \mathrm{a} 0$

[45] D. Zhou, E. Semones, D. O’Sullivan, N. Zapp, M. Weyland, G. Reitz, T. Berger and E. R. Benton, "Radiation Measured for MATROSHKA-1 Experiment with Passive Dosimeters," Acta Astronautica, Vol. 66, No. 1-2, 2010 , pp. 301-308. doi:10.1016/j.actaastro.2009.06.014

[46] E. R. Benton, E. V. Benton, A. L. Frank and M. F. Moyers, "Characterization of the Radiation Shielding Properties of US and Russian EVA Suits Using Passive Detectors," Radiation Measurements, Vol. 41, No. 9-10, 2006, pp. 1191-1201. doi:10.1016/j.radmeas.2006.04.030

[47] E. R. Benton and E. V. Benton, "Space Radiation Dosimetry in Low-Earth Orbit and Beyond," Nuclear Instruments and Methods in Physics Research Section B: Beam Interactions with Materials and Atoms, Vol. 184, No. 1-2, 2001, pp. 255-294. doi:10.1016/S0168-583X(01)00748-0

[48] J. L. Pinfold, "Searching for the Magnetic Monopole and Other Highly Ionizing Particles at Accelerators Using Nuclear Track Detectors," Radiation Measurements, Vol. 44, No. 9-10, 2009, pp. 834-839. doi:10.1016/j.radmeas.2009.10.062

[49] G. Sher, M. Rana and M. Shahzad, "Lead Projectile Fragmentation on Aluminum Target: Improved Experimental Results," World Journal of Nuclear Science and Technology, Vol. 1, 2011, pp. 13-19. doi:10.4236/wjnst.2011.11003

[50] S. Eman, S. Nageeb and A. El-Sersy, "U and Th Determination in Natural Samples Using CR-39 and LR-115 Track Detectors," World Journal of Nuclear Science and Technology, Vol. 2, 2012, pp. 36-40. doi:10.4236/wjnst.2012.21006

[51] M. Abd-Elzaher, M. Badawi, A. El-Khatib and A. Thabet, "Determination of Full Energy Peak Efficiency of $\mathrm{NaI}(\mathrm{Tl})$ Detector Depending on Efficiency Transfer Principle for Conversion Form Experimental Values," World Journal of Nuclear Science and Technology, Vol. 2, 2012, pp. 65-72. doi:10.4236/wjnst.2012.23011

[52] R. Pleskac, et al, "The FIRST Experiment at GSI," $N u$ clear Instruments and Methods in Physics Research Section A: Accelerators, Spectrometers, Detectors and Associated Equipment, Vol. 678, 2012, pp. 130-138. doi:10.1016/j.nima.2012.02.020

[53] B. Braunn, et al., "Nuclear Reaction Measurements of 95 $\mathrm{MeV} / \mathrm{u}{ }^{12} \mathrm{C}$ Interactions on PMMA for Hadrontherapy," Nuclear Instruments and Methods in Physics Research Section B: Beam Interactions with Materials and Atoms, Vol. 269, No. 22, 2011, pp. 2676-2684. doi:10.1016/j.nimb.2011.08.010 